\title{
Electrochemical Detection of Tumor-Derived Extracellular Vesicles on Nanointerdigitated Electrodes
}

\author{
Dilu G. Mathew, ${ }^{\dagger, \nabla}$ Pepijn Beekman, ${ }^{\ddagger, \S, \nabla \odot ~ S e r g e ~ G . ~ L e m a y, ~}{ }^{\Perp}$ Han Zuilhof, ${ }^{\ddagger, L, \#}$ Séverine Le Gac, ${ }^{*,}$ \\ and Wilfred G. van der Wiel ${ }^{*} \dagger \odot$
}

${ }^{\dagger}$ NanoElectronics Group, MESA+ Institute for Nanotechnology, University of Twente, P.O. Box 217, Enschede, 7500 AE The Netherlands

${ }^{\ddagger}$ Laboratory for Organic Chemistry, Wageningen University, Stippeneng 4, Wageningen, 6708WE The Netherlands

${ }^{\S}$ Applied Microfluidics for BioEngineering Research, MESA+ Institute for Nanotechnology, TechMed Center, University of Twente, P.O. Box 217, Enschede, 7500 AE The Netherlands

"Bioelectronics, MESA+ Institute for Nanotechnology, University of Twente, P.O. Box 217, Enschede, 7500 AE The Netherlands

${ }^{\perp}$ School of Pharmaceutical Sciences and Technology, Tianjin University, Tianjin, 300072 China

\# Department of Chemical and Materials Engineering, King Abdulaziz University, Jeddah, 21589 Saudi Arabia

Supporting Information

ABSTRACT: Tumor-derived extracellular vesicles (tdEVs) are attracting much attention due to their essential function in intercellular communication and their potential as cancer biomarkers. Although tdEVs are significantly more abundant in blood than other cancer biomarkers, their concentration compared to other blood components remains relatively low. Moreover, the presence of particles in blood with a similar size as that of tdEVs makes their selective and sensitive detection further challenging. Therefore, highly sensitive and specific biosensors are required for unambiguous tdEV detection in complex biological environments, especially for decentralized point-of-care analysis. Here, we report an electrochemical

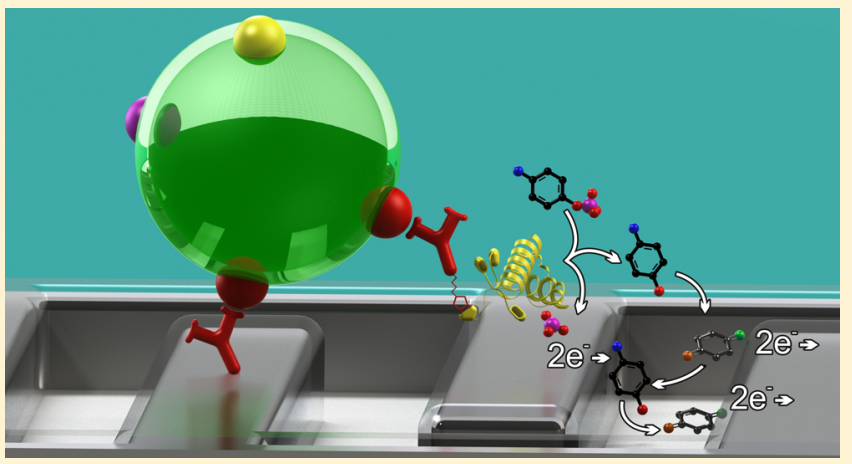
sensing scheme for tdEV detection, with two-level selectivity provided by a sandwich immunoassay and two-level amplification through the combination of an enzymatic assay and redox cycling on nanointerdigitated electrodes to respectively enhance the specificity and sensitivity of the assay. Analysis of prostate cancer cell line tdEV samples at various concentrations revealed an estimated limit of detection for our assay as low as 5 tdEVs/ $\mu \mathrm{L}$, as well as an excellent linear sensor response spreading over 6 orders of magnitude $\left(10-10^{6}\right.$ tdEVs $\left./ \mu \mathrm{L}\right)$, which importantly covers the clinically relevant range for tdEV detection in blood. This novel nanosensor and associated sensing scheme opens new opportunities to detect tdEVs at clinically relevant concentrations from a single blood finger prick.

KEYWORDS: Nanoelectrodes, redox cycling, enzymatic amplification, tumor-derived extracellular vesicles, microfluidics

\begin{abstract}
$\mathrm{L}$ iquid biopsies are highly promising for metastatic cancer $\checkmark$ disease management. In this noninvasive approach, a sample of blood (typically a few $\mathrm{mL}$, e.g., from a finger prick) is screened for the presence of tumor biomarkers, such as circulating tumor DNA (ctDNA), miRNAs, tumor-derived extracellular vesicles (tdEVs), or circulating tumor cells (CTCs). ${ }^{2}$ It has been established that, compared to imaging techniques (magnetic resonance imaging (MRI) in conjunction with computed tomography (CT)), CTC quantification in liquid biopsies has a better prognostic value, ${ }^{1,3}$ while being significantly less demanding from a clinical point-of-view: the procedure is much more patient-friendly, cheaper and does not require any administration of toxic contrast agents. Furthermore, blood analysis can be repeated at higher frequency (e.g., a few times per month vs a few times per year for MRI), while allowing close monitoring of a patient's response to therapy.
\end{abstract}

However, the main challenge to be overcome in this approach is the extremely low relative concentration of CTCs. Moreover, for every CTC (typically <10 CTCs per $\mathrm{mL}$ of blood), there are millions of white blood cells and billions of red blood cells. In contrast to CTCs, tdEVs, which are constantly released by tumor cells in blood, occur at a much higher concentration (10-10 $0^{6}$ EVs per $\mu \mathrm{L}$ of blood). ${ }^{4}$ Extracellular vesicles (EVs) are nanometer-sized ( $30 \mathrm{~nm}$ to $1 \mu \mathrm{m}$ ) particles, enclosed by a phospholipid bilayer membrane and containing a great variety of biological molecular information on their cells and/or tissues of origin. ${ }^{5-7}$ EVs are shed by all cell types and found in

Received: July 5, 2019

Revised: September 4, 2019

Published: September 19, 2019 


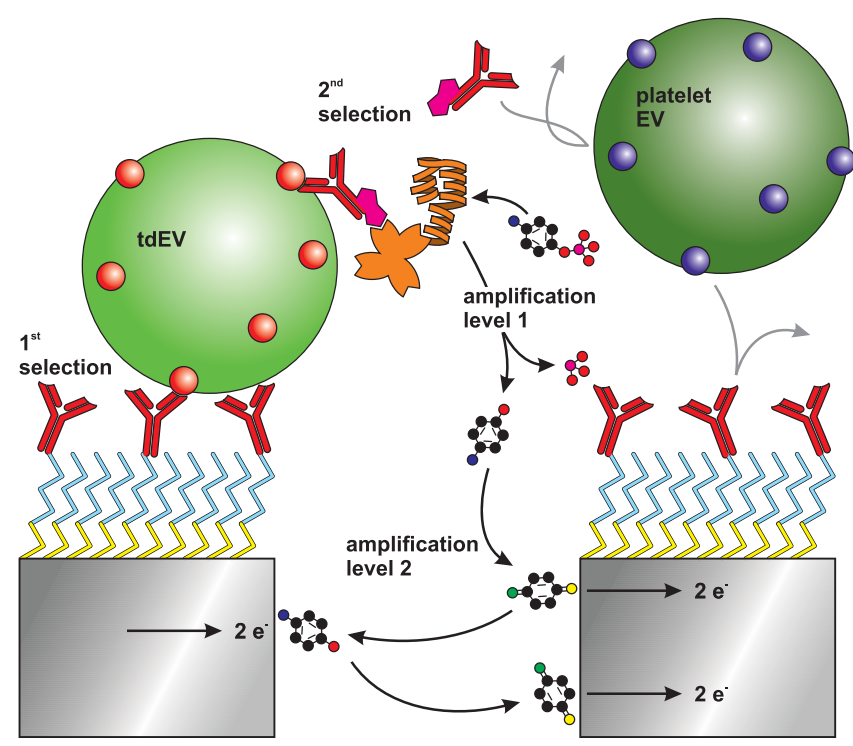

\begin{tabular}{|c|c|}
\hline & $\begin{array}{l}\text { Streptavidin-conjugated } \\
\text { Alkaline phosphatase } \\
\text { (SAV-ALP) }\end{array}$ \\
\hline & $\begin{array}{l}\text { Reporter } \\
\text { anti-EpCAM (R-AE) }\end{array}$ \\
\hline & $\begin{array}{l}\text { Capture } \\
\text { anti-EpCAM (C-AE) }\end{array}$ \\
\hline & $\begin{array}{l}\text { p-aminophenyl phosphate } \\
\text { (pAPP) }\end{array}$ \\
\hline & p-aminophenol (pAP) \\
\hline & p-quinone imine (pQI) \\
\hline & Generic marker \\
\hline & $\begin{array}{l}\text { Epithelial cell adhesion } \\
\text { molecule (EpCAM) }\end{array}$ \\
\hline & $\begin{array}{l}\text { poly(ethylene glycol) } \\
\text { diglycidyl ether (PEGDGE) }\end{array}$ \\
\hline & $\begin{array}{l}\text { amino } \\
\text { undecanethiol (AUT) }\end{array}$ \\
\hline & Pt nanoelectrode \\
\hline
\end{tabular}

Figure 1. Schematic illustration of tdEV sensing using a sandwich immunoassay and redox cycling on nIDEs resulting in a two-level selectivity and a two-level amplification. tdEVs are captured using C-AE tethered to electrodes (first level of selectivity). The binding of R-AE to the tdEVs completes the antibody-antigen-antibody sandwich (second-level selectivity), after which the enzyme ALP is introduced using a biotin-SAV interaction. ALP provides an enzymatic amplification of pAPP to pAP by substrate cleavage (first-level amplification), which is followed by an electrochemical signal amplification via the oxidation of $\mathrm{pAP}$ to $\mathrm{pQI}$ and subsequent redox cycling thereof between the nIDE electrodes (secondlevel amplification).

all bodily fluids, where they play an important role in (inter)cellular communication. ${ }^{8,9}$ All EVs share the same generic EV-membrane protein repertoire, e.g., CD9, CD63, and CD81 being present on the vast majority of blood cellderived EVs. ${ }^{10,11}$ Next to this, tdEVs exhibit membrane proteins that are specific of their cellular origin, e.g., cancer biomarkers HER2, EGFR, and epithelial cell adhesion molecule (EpCAM). 1,10,12 Notably, EpCAM has been widely used for the isolation and detection of both CTCs and tdEVs, ${ }^{1}$ which are found in blood from the early stages of cancer to metastasis. The concentration of both CTCs and tdEVs increases with the progression of the tumor. ${ }^{13-16}$ The widespread tdEV concentrations naturally occurring in blood make them promising alternative cancer biomarkers. The variation between patients with low and high tdEV abundance is relatively much higher than for CTCs (a factor of $10^{6}$ for EVs vs. a factor of maximally $\sim 10^{3}$ for CTCs), and therefore statistically more unlikely to yield false-negative results.

However, before tdEVs can be considered in clinical routines and liquid biopsy analysis, reliable, unambiguous, highly sensitive and specific methods must be developed for their isolation, detection, and quantification in complex matrices such as blood. Species in the EV size range are difficult to characterize using existing analytical techniques suitable to single molecules or cells, which are, respectively, smaller and larger than EVs. Furthermore, blood comprises various other entities in the same size range as EVs and often present with much higher concentrations, such as protein aggregates, lipoproteins, cell debris and, most notably, noncancerous EVs, from which tdEVs need to be unequivocally distinguished. ${ }^{17}$ EVs are often studied using flow cytometry and/or fluorescence microscopy. ${ }^{18-24}$ Although these techniques provide unique molecular information on tdEVs, they often lack the sensitivity and/or resolution required to detect both the rarest and/or smallest tdEVs. These techniques also require substantial sample volumes. In contrast, single EVs can be detected using atomic force microscopy (AFM), ${ }^{25}$ nanoparticle tracking analysis (NTA), ${ }^{26}$ resistive pulse sensing, ${ }^{26}$ Raman spectroscopy ${ }^{27,28}$ or a combination of some of these techniques, whose throughput and level of technicality is however too low for practical medical/clinical use.

Altogether there is a clear need to be able to detect tdEVs at concentrations as low as $1-100$ per $\mu \mathrm{L}$ and across an extended clinically relevant concentration range. ${ }^{4}$ However, only a few endeavors have led to the development of sensors sensitive enough to detect such low concentrations. Recently, Zhang et al. $^{29}$ reported immunocapturing of tdEVs on antibodymodified herringbone in microfluidic channels followed by their detection using fluorescence microscopy, after amplification of the signal using an enzymatic reaction. Although they reported a limit of detection (LOD) of $10 \mathrm{EVs} / \mu \mathrm{L}$, the signal was barely distinguishable from the background, and their approach worked over a linear detection range from $10-10^{3}$ $\mathrm{EVs} / \mu \mathrm{L}$. Using amperometric detection of enzymatic activity after magnetic immuno-enrichment with nanocubes, Boriachek et al. ${ }^{30}$ analyzed EVs from placental cells using placental alkaline phosphatase as a marker. Their reported LOD was as low as $1 \mathrm{tdEV} / \mu \mathrm{L}$. However, again, the linear range of their assay only covered a $1-10^{4} \mathrm{EVs} / \mu \mathrm{L}$ concentration range. Huang et al. ${ }^{31}$ developed an electrochemical detection platform using aptamers as detection probes and a combination of hemin/G-quadruplex DNAzyme-peroxidase reaction and complex rolling circle amplification to achieve signal amplification. Although they achieved a detection limit of $\sim 1 \mathrm{tdEV} / \mu \mathrm{L}$, they as well had a narrow linear range of detection from 1 to $10^{3} \mathrm{EVs} / \mu \mathrm{L}$, making this assay less versatile for clinical applications.

Here, we report an ultrasensitive tdEV detection assay at clinically relevant concentrations using a double amplification mechanism combining redox cycling and an enzymatic reaction, as well as a sandwich immunoassay ensuring a two- 
$\mathbf{a}$

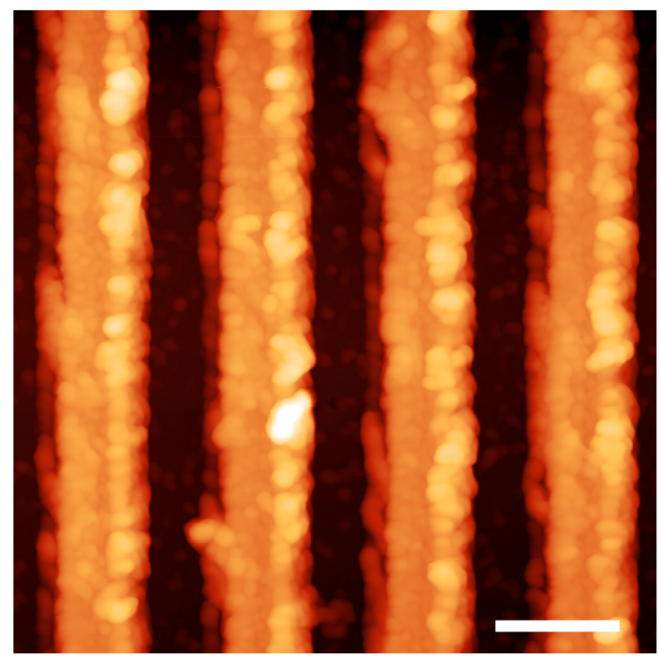

b

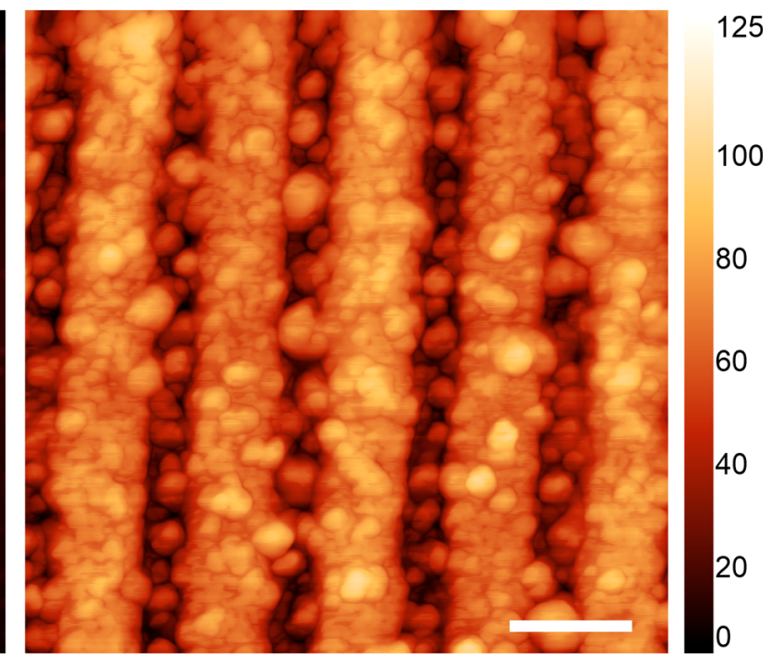

Figure 2. Atomic force microscope height images: (a) bare electrodes before chemical modification and (b) after modification and capture of EpCAM-positive tdEVs derived from LnCAP cell lines on nIDEs. The captured objects are $30-150 \mathrm{~nm}$ in diameter, which is in good agreement with small EV dimensions (scale bar: $200 \mathrm{~nm}$ ).

level selectivity. The assay is implemented in a lab-on-a-chip format allowing the analysis of small sample amounts, in the (low) microliter range. Uniquely, the linear dynamic range achieved with our assay spanning 6 orders of magnitude largely overlaps with the range of tdEV concentrations naturally occurring in cancer patient blood. Using tdEVs obtained from the cell culture medium, we experimentally demonstrated a LOD of $10 \mathrm{tdEVs} / \mu \mathrm{L}$ well above the background signal in our assay and extrapolated a theoretical LOD as low as a 5 tdEVs/ $\mu \mathrm{L}$ from the established calibration curve. Compared to previously reported methods, our antifouling coating in combination with our 2 -fold selective scheme awards excellent specificity for tdEV detection compared to EVs of other origins, as demonstrated here using platelet-derived EVs (giving 60 times less signal at a $10^{2}$-fold higher concentration).

\section{SANDWICH IMMUNOASSAY ON NANOSCALE INTERDIGITATED ELECTRODES}

The detection principle of our assay is illustrated in Figure 1. To achieve an amplification level that is powerful enough to detect tdEVs at physiologically relevant concentrations, we use a two-level amplification strategy: (i) a first enzymatic amplification using alkaline phosphatase (ALP), releasing electrochemically active species, followed by (ii) electrical signal amplification via electrochemical redox cycling on nanoscale interdigitated electrodes (nIDEs). Given the complexity of the targeted biological sample, exquisite selectivity is required to get a signal that solely arises from the presence of tumor-derived species, i.e., with a low background signal. Here, a sandwich immunoassay with tumor-specific antibodies is implemented, providing a twolevel selectivity. The assay comprises a capture anti-EpCAM (C-AE) antibody that captures tdEVs and a reporter antibody (anti-EpCAM, R-AE) conjugated to ALP through biotinstreptavidin interactions. Here, the same antibody clone is employed for the two steps of the immune-affinity assay, VU1D9, which is an anti-EpCAM clone proven to be stable and to have high affinity for EpCAM $\left(K_{\mathrm{d}} \sim 2.7 \times 10^{-10} \mathrm{M}\right){ }^{32}$ Tethering of $\mathrm{C}-\mathrm{AE}$ on the electrodes involves three steps. First, amine-terminated thiol (amino-undecanethiol, AUT) is selfassembled on the Pt electrodes. Second, an amine-reactive bifunctional poly(ethylene glycol) diglycidyl ether (PEGDGE) is reacted with the AUT layer to add an antifouling linker layer. Subsequently, the C-AE antibody is covalently linked under mild conditions at a slightly basic $\mathrm{pH}$ (8.3) and high ionic strength ( $2 \mathrm{M}$ sodium phosphate). ${ }^{33}$ This $\mathrm{C}-\mathrm{AE}$ enables the specific capture of tdEVs derived from EpCAM-expressing human prostate adenocarcinoma cell lines ( $\mathrm{LNCaP}$ ) and provides as such the first level of selectivity. After their capture, the tdEVs interact with biotinylated reporter anti-EpCAM antibodies (R-AE), to provide the second level selectivity, since noncancerous EVs (e.g., blood cell-derived) would not be recognized by either the C-AEs or the R-AEs. The use of the same antibody clones for both immuno-affinity steps can lead to the partial dissociation of the antibody/antigen complex formed between the C-AE and EVs to form a new antibody/ antigen complex with $\mathrm{R}-\mathrm{AE}$, releasing thereby the EVs from the surface. Using a distinct pair of antibodies as $\mathrm{C}-\mathrm{AE}$ and $\mathrm{R}-\mathrm{AE}$ (e.g., VU1D9 and HO-3) would be more favorable in this context. However, considering it may be entropically unfavorable for R-AE to approach the $\mathrm{C}-\mathrm{AE} /$ antigen complex and because each EV should be captured through multiple antigen/antibody interactions, it seems very unlikely that EVs are released during the second immune-affinity step. The biotin moiety of R-AE next interacts with streptavidinconjugated alkaline phosphatase (SAV-ALP). ALP is wellknown for its ability to cleave substrates containing phosphate groups. Here, the substrate (para-aminophenyl phosphate, pAPP) is chosen since its uncleaved form is electrochemically inert, while its cleaved form, para-aminophenol (pAP), is electrochemically active ${ }^{34}$. This enzymatic reaction provides the first amplification mechanism. During electrochemical measurements, one of the working electrodes of the nIDE is kept at the reduction potential (a potential well below the formal potential) of pAP, while the second working electrode is swept from a potential below to a potential above its formal potential. At the anode, pAP is oxidized into para-quinone imine (pQI), which diffuses toward the cathode. At the 


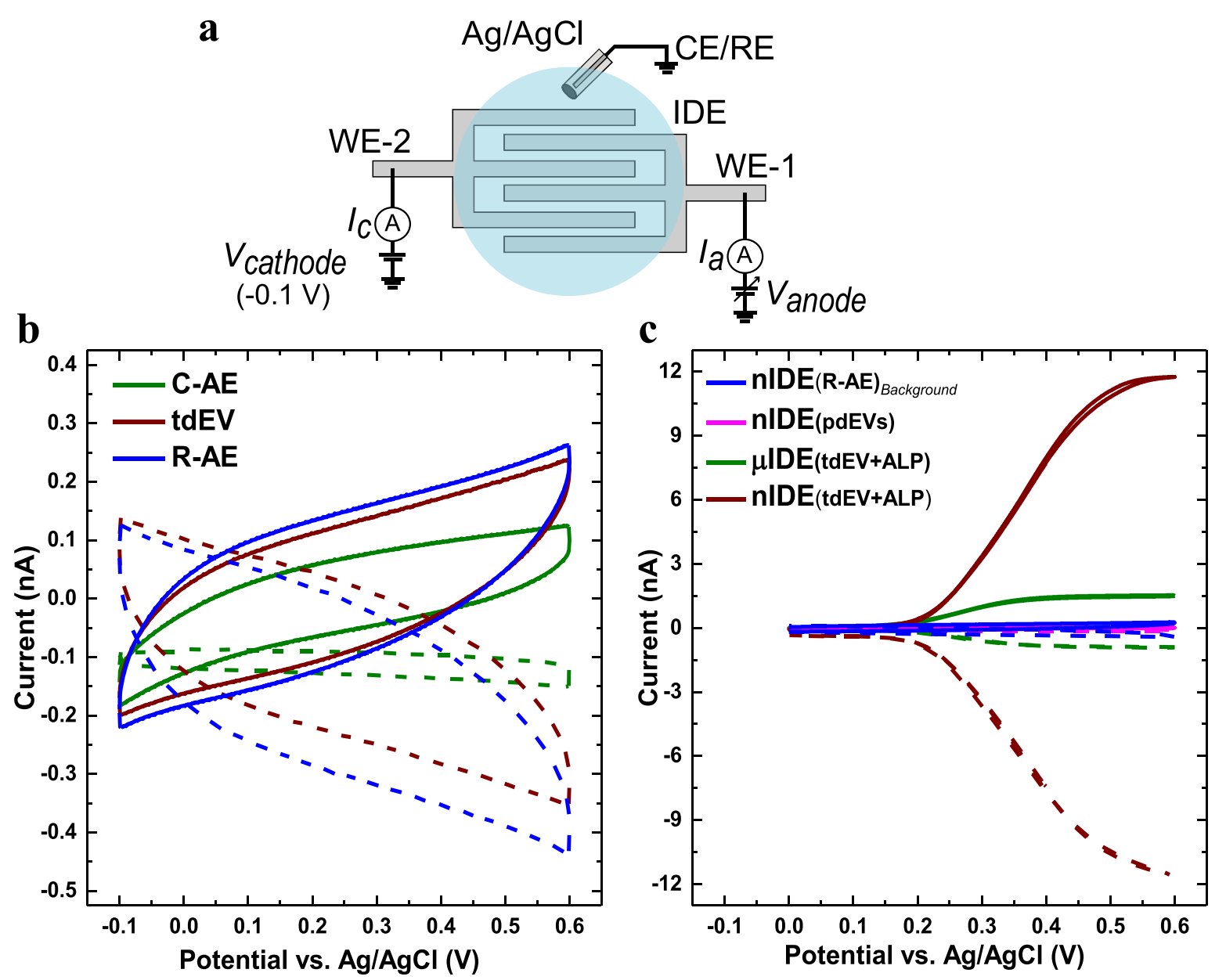

Figure 3. Cyclic voltammograms of tdEVs on nIDEs: evaluation of the device specificity. (a) Schematic representation of the electrochemical measurement setup. Cyclic voltammograms (CVs) recorded (b) after various steps of functionalization of the nIDEs, after C-AE surface modification (green), after tdEV capture (brown), and after formation of a sandwich with R-AE (blue), and (c) in the presence of tdEVs on nIDEs (brown) and on $\mu \mathrm{IDEs}$ (green), or in the presence of pdEVs on nIDEs (magenta). The background signal (blue) corresponds to a device after the antibody sandwich formation. The currents $I_{\text {anode }}$ (solid lines) and $I_{\text {cathode }}$ (dashed lines) were measured at the anode and the cathode set of electrodes of the nIDEs/ $\mu$ IDEs, respectively. CVs were acquired for a $1 \mathrm{mM}$ pAPP solution in PBS ( $\mathrm{pH} 7.4)$ between $-0.1 \mathrm{~V}$ and $+0.6 \mathrm{~V}$ vs Ag/ $\mathrm{AgCl}$ at a scan rate of $50 \mathrm{mV} / \mathrm{s}$.

cathode, $\mathrm{pQI}$ is reduced back into pAP. The electrochemical reaction can be denoted as $\mathrm{pAP} \leftrightarrows \mathrm{pQI}+2 e^{-}$. Since the gaps between the electrodes of the nIDEs are small $(120 \mathrm{~nm})$, the pAP and PQI molecules continuously and efficiently shuttle between the two working electrodes via diffusion, producing a steady-state current, which directly scales with the tdEV concentration. This redox cycling provides the second level of amplification.

The nIDEs comprised two sets of interdigitated nanoelectrode arrays (100 nm width, $30 \mu \mathrm{m}$ length, $120 \mathrm{~nm}$ spacing, and $70 \mathrm{~nm}$ height) defined by electron-beam lithography and platinum evaporation. The narrow spacing between the electrodes tremendously enhanced the redox cycling performance, while still allowing the capture of the smallest (most abundant) EVs in the void between the electrodes. ${ }^{35}$ EVs larger than $120 \mathrm{~nm}$ can be captured on the top surface of the electrodes and can simply contribute to the signal following the same mechanism.

The sensing area of the nIDEs was embedded in a poly(dimethylsiloxane) (PDMS) microfluidic channel $(0.2 \times$ $\left.3 \times 6 \mathrm{~mm}^{3}\right)$ to facilitate the exchange of reagents by simple micropipetting directly through the channel inlet during surface modification and electrochemical measurements (see
Supporting Information, Figure SI-1). ${ }^{36}$ A pipette tip was installed in the outlet of the microfluidic device as a collection reservoir facilitating the back and forth injection of the sample using a pipette and handling volumes larger than the microchannel itself. After the introduction of every new component, a washing step was implemented by injecting 100 $\mu \mathrm{L}$ of phosphate-buffered saline (PBS).

tdEV Detection on nIDEs. tdEVs were prepared following the protocols described in SI-2. In brief, tdEVs were isolated from LNCaP cells cultured in serum-free medium. The tdEV samples were first characterized using Nanoparticle Tracking Analysis (NTA) to estimate the tdEV concentration (i.e., $10^{6}$ \% $\mu \mathrm{L}$ ). The surface functionalization of the electrodes and $\mathrm{tdEV}$ capture steps were both evaluated using AFM. AFM images acquired on a functionalized unpatterned (plain) Pt surface are presented in Figure SI-2 (see Supporting Information). EV capture was next validated on patterned (nIDE) devices using AFM. A sample of LNCaP-derived tdEVs $(25 \mu \mathrm{L}$, concentration of $10^{6} \mathrm{EVs} / \mu \mathrm{L}$, as determined by NTA), was incubated on the C-AE-functionalized electrode surface. As depicted in Figure 2, which presents AFM images before and after the capture of tdEVs on C-AE functionalized electrodes, circular objects were found on the electrodes, with an estimated 
diameter of $30-150 \mathrm{~nm}$. These objects correspond to relatively small EVs, ${ }^{26}$ which are the least susceptible to shear forces when flushing the microchannel to remove unbound species, since the Stokes drag force linearly scales with the object size (while neglecting viscous deformation or size-dependence of the affinity). The system was not intentionally designed to exclude larger tdEVs, since EVs captured on the nIDEs were not expected to short-circuit the electrodes due to their dielectric properties. It may be that tdEVs are captured in the space between the electrodes, which is not modified with the antifouling layer and the antibodies. These objects could be captured through antigen/antibody interactions by antibodies present on the sidewalls of the electrodes. Alternatively, some tdEVs could be nonspecifically bound on the surface. The presence of the latter EVs does not influence the outcome of the measurements, which directly depends on the interactions with the second antibody (second level of selectivity).

After validation of the surface chemistry on plane substrates (see SI-4), the same functionalization protocol was applied on nIDEs before electrochemical measurements. $100 \mu \mathrm{L}$ of a tdEV solution in PBS was injected into the microfluidic channel, and this solution was flushed back and forth multiple times during the incubation for $90 \mathrm{~min}$. Different concentrations in EVs (initially $10^{6}$ EVs per $\mu \mathrm{L}$ ) were tested to study the concentration-dependent response of our nanosensor and associated sensing assay. Following this, the microchannel was flushed with a PBS solution to remove unspecifically adsorbed particles on the electrodes. Consequently, the IDEs were incubated with the biotinylated R-AE, $(10 \mu \mathrm{L}, 25 \mathrm{mg} / \mathrm{mL}$ in PBS, 30 min incubation) and washed with PBS. Subsequently, SAV-ALP was introduced $(10 \mu \mathrm{L}, 10 \mathrm{U}$ solution in PBS, 30 min incubation) to interact with the biotin on the R-AE. Next, the IDEs were washed with PBS and incubated with a pAPP solution (100 $\mu \mathrm{L}, 10 \mathrm{mM}$ in PBS, $45 \mathrm{~min}$ incubation), before electrochemical measurements were started. It should be noted that while the performance of ALP is optimal under alkaline conditions, a physiological $\mathrm{pH}$ is preferred for handling EVs. Consequently, the ALP incubation step was performed in PBS $(\mathrm{pH} 7.4)$ at room temperature, without active temperature control. Negative control measurements were performed using platelet-derived EVs (pdEV) at $10^{8} \mathrm{pdEVs} / \mu \mathrm{L}$, which are not EpCAM positive, and do not interact as such with antiEpCAM antibodies (both $\mathrm{C}-\mathrm{AE}$ and $\mathrm{R}-\mathrm{AE}$ ). Finally, to evaluate the amplification and thereby gain in sensitivity provided by the nanoscale electrodes, their performance was compared to that of microscale IDEs ( $\mu$ IDEs), which were 3 $\mu \mathrm{m}$ wide, $70 \mathrm{~nm}$ high and spaced by $3 \mu \mathrm{m}$. The height and total sensing area of sets of electrodes were kept the same for both devices to facilitate the performance comparison.

The schematic representation of the measurement setup is provided in Figure 3a; it includes two sets of independent working electrodes (WE-1 and WE-2) and an external Ag/ $\mathrm{AgCl}$ reference electrode (RE). A fixed potential $(-0.1 \mathrm{~V})$ was applied to WE-2 with respect to RE. Since the RE current is very low, the stability of RE was not compromised, even when there was no additional counter electrode (CE). Hence, the $\mathrm{CE}$ terminal of the potentiostat was connected to RE. The voltammetric responses (scan rate of $50 \mathrm{mV} / \mathrm{s}$ ) of the nIDEs were first recorded after different steps of functionalization, i.e., (1) after C-AE functionalization, (2) after tdEV capture, (3) after R-AE immobilization, and (4) after conjugation of the biotinylated R-AE to SAV-ALP and subsequent washing, as well as in the presence of pdEVs instead of tdEVs (negative control).

Figure $3 \mathrm{~b}$ presents typical cyclic voltammograms obtained after the different surface functionalization steps, yet before the introduction of ALP on the surface. In all three cases considered here, no significant change in the recorded current was observed when pAPP was added in the solution, and a maximum current of $262 \mathrm{pA}$ was recorded at $0.6 \mathrm{~V}$ vs. $\mathrm{Ag} /$ $\mathrm{AgCl}$ after addition of the R-AE. Since pAPP is electrochemically inactive, no redox activity is expected, as observed here. Noteworthy, the recorded voltammograms are similar to the I/ $V$ characteristics of an $\mathrm{RC}$ series circuit, with capacitive charging and discharging upon voltage sweeping, and a hysteresis. Furthermore, a change in the maximal amplitude at $0.6 \mathrm{~V}$ vs. $\mathrm{Ag} / \mathrm{AgCl}$ was observed after each surface functionalization step. In particular, a considerable capacitive change was found after the immobilization of the tdEVs, which can be noticed with the change in the maximal current value (at $0.6 \mathrm{~V}$ vs. $\mathrm{Ag} / \mathrm{AgCl}$ ) from $125.1 \pm 6.5 \mathrm{pA}$ to $245.1 \pm 28.3$ $\mathrm{pA}$ after the immobilization. Although the capacitive change after the formation of the sandwich assay with R-AE is discernible, it is relatively low compared to the preceding surface functionalization step with $10^{6} \mathrm{tdEVs} / \mu \mathrm{L}$ (from 245.1 $\pm 28.3 \mathrm{pA}$ to $267.6 \pm 15 \mathrm{pA})$. This behavior might be indicative of capacitive charging through the vesicles. On the sample with negative controls (with $10^{8} \mathrm{pdEVs} / \mu \mathrm{L}$ ), as discussed below, this capacitance change was however not observed, corroborating this argument. The contrast between the signals recorded for our positive and negative control samples also indicates that the antifouling layer plays an essential role in our device, and has performed as expected.

In a following step, we compared the response of nIDEs and $\mu \mathrm{IDEs}$, using similar conditions as before (10 $\mu \mathrm{L}, 10^{6}$ tdEVs/ $\mu \mathrm{L}, 1 \mathrm{mM}$ pAPP in PBS ( $\mathrm{pH} 7.4)$, scan rate of $50 \mathrm{mV} / \mathrm{s}$ ), after the introduction of the SAV-ALP. Figure $3 \mathrm{c}$ presents characteristic sigmoidal curves of diffusion-limited redox cycling currents on closely spaced working electrodes. The limiting current of nIDEs increases with decreasing the gap size between the electrodes. Therefore, although the total sensing surface area of the nIDEs and $\mu$ IDEs was the same, the $3-\mu \mathrm{m}$ gap between the $\mu \mathrm{IDEs}$ resulted in a significantly lower limiting current $(1.53 \pm 0.01 \mathrm{nA})$ compared to the nIDEs, which were separated by $120 \mathrm{~nm}(11.76 \pm 0.04 \mathrm{nA})$. Furthermore, the collector efficiency (ratio of cathode-to-anode limiting currents) of $\mu \mathrm{IDEs}$ was found to be only $62.3 \%$ compared to $99.8 \%$ for the nIDEs. This significant difference indicates that the redox mediator molecules cycle fewer times between the anode and the cathode for the $\mu$ IDEs before diffusing into the bulk solution. Altogether, the nanoscale electrodes provided an $\sim 8$ times larger amplification of the signal than their microscale counterparts.

Next, we investigated the specificity of our device for the capture and analysis of tdEVs (Figure 3c). tdEVs and pdEV samples were analyzed under the same conditions as before. We compared the response of (1) tdEV+R-AE (blue curve), but before the incubation with SAV-ALP on nIDEs, (2) pdEV on nIDEs (magenta curve), (3) tdEV on nIDEs (brown curve), and (4) tdEV on $\mu$ IDEs (green curve), after introduction of all required reagents for the assay. The capacitive current $(267.6 \pm 15 \mathrm{pA})$ recorded for the tdEV+R$\mathrm{AE}$ sample $\left(10^{6}\right.$ particles $\left./ \mu \mathrm{L}\right)$ was higher than for the pdEV sample $(170.1 \pm 13.2 \mathrm{pA})$ at $0.6 \mathrm{~V}$ for $\sim 10^{8}$ particles $/ \mu \mathrm{L}$. Moreover, comparing the limiting currents, $\sim 60$ times 
a

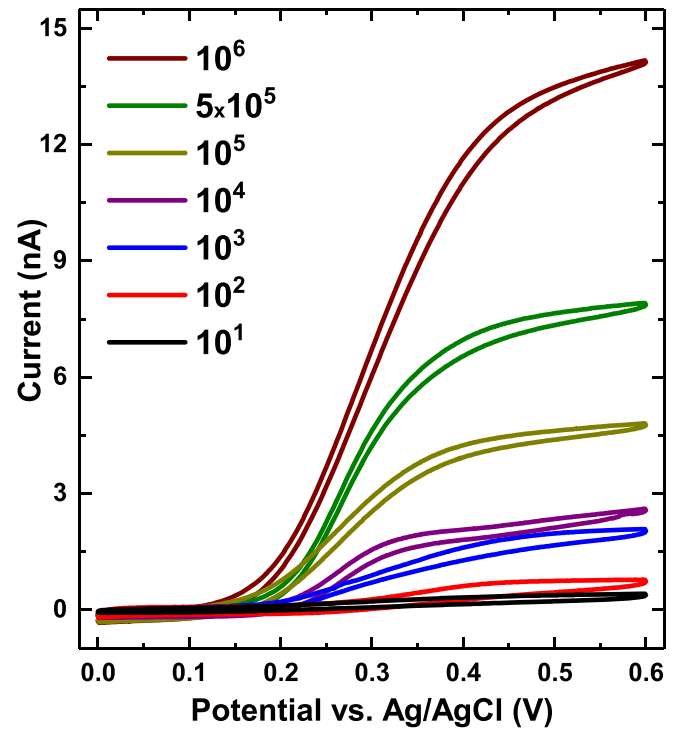

b

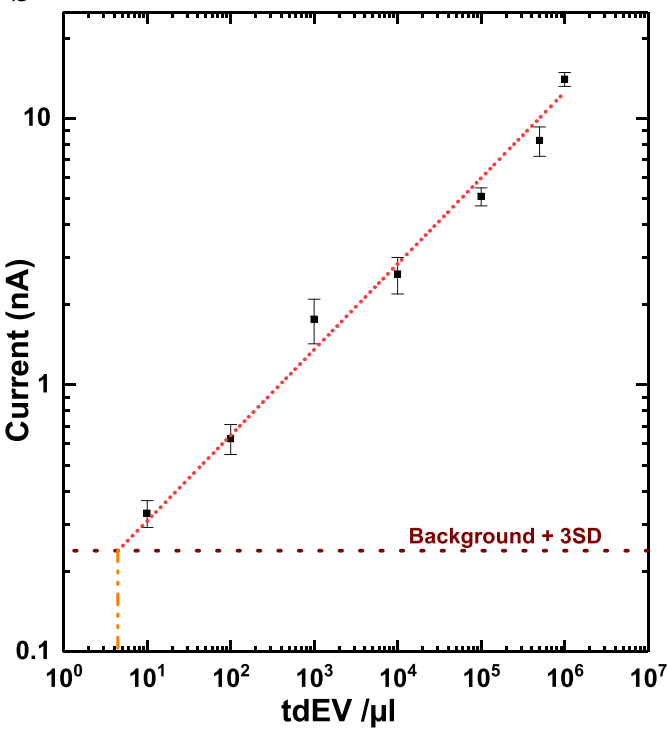

Figure 4. Sensitivity and dynamic range of the assay for the detection of tdEVs. (a) Anodic cyclic voltammograms recorded at the anode for tdEV samples with concentrations ranging from 10 to $10^{6}$ tdEVs per $\mu \mathrm{L}$. (b) Associated calibration curve (based on the limiting currents recorded at 0.6 $\mathrm{V}$ vs $\mathrm{Ag} / \mathrm{AgCl}$ ) revealing a dynamic range spanning at least 6 orders of magnitude (number of devices, $n=3$ ). The horizontal dotted line depicts the background level plus three times the standard deviation (SD) of the redox current; from this horizontal line and the calibration curve, a theoretical LOD as low as $5 \mathrm{tdEVs} / \mu \mathrm{L}$ is found. (Conditions: $1 \mathrm{mM}$ pAPP solution in PBS (pH 7.4); scan rate of $50 \mathrm{mV} / \mathrm{s}$.)

amplification in signal was observed compared to the control sample with pdEV for nIDE devices (magenta curve). Again, these results collectively suggest that tdEVs are specifically captured on the electrode surface, while pdEVs are not, illustrating the specificity of the nanosensor.

As a next step, the sensitivity and dynamic range of the assay were evaluated, through serial dilution of the initial tdEV sample on three different devices $(n=3)$. For each device and for each concentration, $\mathrm{CV}$ measurements were performed as before, with $1 \mathrm{mM}$ pAPP in PBS buffer at $\mathrm{pH} 7.4$ with a scan rate of $50 \mathrm{mV} / \mathrm{s}$. This recording was repeated three times with a 5 min interval to demonstrate the stability of the measurements. The CVs recorded after varying the tdEV concentration between 10 and $10^{6} \mathrm{EVs}$ per $\mu \mathrm{L}$ are presented in Figure $4 a$, showing a significant influence of the tdEV concentration. From these data recorded using three independent devices, a calibration curve was established (Figure $4 \mathrm{~b}$ ) using the limiting current at $0.6 \mathrm{~V}$ revealing an excellent linear dynamic range spanning at least 6 orders of magnitude and successful measurements at least down to 10 $\mathrm{tdEVs} / \mu \mathrm{L}$, with a readout distinctively above the background signal. By extrapolating the slope of this calibration curve, the current LOD for our assay was evaluated to be ca. $5 \mathrm{tdEVs} / \mu \mathrm{L}$. In conclusion, we report a novel electrochemical biosensor and associated measurement principle for the highly selective, highly sensitive, and robust quantification of tumor-derived extracellular vesicles. For this, we used a two-level amplification of the signal and a two-level specificity. High assay sensitivity was attained through enzymatic amplification combined with redox cycling between nanoscale interdigitated electrodes. In addition, the high specificity was achieved from the presence of two independent selection steps in the sandwich immunoassay. Using the herein reported device and sensing protocol, we have reached a very high sensitivity that is clinically relevant for the detection of tdEVs, with a measured LOD as low as 10 tdEVs per $\mu \mathrm{L}$, while extrapolation of the calibration curve suggests a projected LOD of $5 \mathrm{tdEVs} / \mu \mathrm{L}$. While having an LOD in the same order of magnitude as the currently most sensitive reported systems, importantly, the detection range largely covers the concentration of tdEVs $\left(10-10^{6} \mathrm{tdEVs} / \mu \mathrm{L}\right)$ found in metastatic cancer patients. Further optimization of the nanosensor and assay performance is ongoing via changes in and stabilization of experimental conditions like $\mathrm{pH}$, incubation time, and operating temperature. Furthermore, the herein used poly(ethylene glycol)-based antifouling layer may not be sufficient when working with samples in more complex media. In that case, the antifouling layer can be adjusted through the incorporation, for instance, of zwitterionic polymer brushes. ${ }^{37}$ Importantly, this technology can be applied to a wide range of (rare) biomarkers by simply incorporating a different recognition element (e.g., a different antibody). Furthermore, this amperometric sensing method has the potential to be developed as a portable point-of-care sensing device, which can also be useful in population-wide disease screening.

\section{MATERIALS AND METHODS}

Materials. Dichloromethane and ethanol (VLSI grade) were purchased from VWR (Amsterdam, The Netherlands). Acetone (VLSI grade), Harris Uni-Core $1 \mathrm{~mm}$ I.D. biopsy punches, sodium phosphate, 11-amino-1-undecanethiol hydrochloride (AUT), (poly(ethylene glycol) diglycidyl ether (PEGDGE), PBS tablets, bovine serum albumin (BSA), and 4-aminophenyl phosphate monosodium salt hydrate (pAPP) were obtained from Merck (Zwijndrecht, The Netherlands). Capturing anti-EpCAM (C-AE), biotinylated reporter antiEpCAM (R-AE), and extracellular vesicles were received as a kind gift from Immunicon corp (Huntingdon Valley, United States). Streptavidin-conjugated alkaline phosphatase (SAVALP) was purchased from Thermo Fisher (Eindhoven, The Netherlands). Sylgard 184 was obtained from Farnell (Utrecht, The Netherlands). Buffers were filtered through a $0.2 \mu \mathrm{m}$ 
syringe filter before use (Whatman, Little Chalfont, United Kingdom).

Device Fabrication. nIDEs and $\mu$ IDEs were fabricated using a combination of optical and electron-beam lithography (EBL) and metal evaporation and lift-off process. First, a 300 $\mathrm{nm} \mathrm{SiO}$ layer was thermally grown on a $10 \mathrm{~cm} \mathrm{Si}(100)$ wafer at $1000{ }^{\circ} \mathrm{C}$. A first optical lithography step was carried out, followed by evaporation of metals ( $\mathrm{Ti}$, adhesion layer, and $\mathrm{Pt}$ ) and lift-off for defining the markers for EBL. Subsequently, nIDEs were patterned using EBL. $\mathrm{CHF}_{3}$-based plasma etching was next performed to recess the adhesive metal layer into the substrate in the following step. After the etching, metal deposition (Ti, $5 \mathrm{~nm}$; Pt, $70 \mathrm{~nm}$ ) and lift-off were done to form the IDEs. Successively, the nIDEs were connected to contact pads via contact leads using a second optical lithography step followed by another metal evaporation (Ti, $5 \mathrm{~nm}$; Pt, $100 \mathrm{~nm}$ ) and lift-off. A $300 \mathrm{~nm}$ layer of parylene-C was evaporated as a first passivation layer. A third optical lithography step was done, and using the patterned photoresist as a mask, $\mathrm{SiO}_{2}(30$ $\mathrm{nm}$ ) was evaporated on top of the parylene-C layer. This oxide layer covered the entire device except for the contact pads and a rectangular window of $30 \times 70 \mu \mathrm{m}^{2}$ over the nIDEs. The two passivation layers (parylene and $\mathrm{SiO}_{2}$ ) prevented current leakage through the contact leads. The fabricated chips were then diced to $25 \times 20 \mathrm{~mm}^{2}$ chips (Disco DAD321 dicing machine). Bare Pt substrates to validate and characterize the surface functionalization were prepared by sputtering a $10 \mathrm{~nm}$ layer of Ta as an adhesive layer followed by a $100 \mathrm{~nm}$ layer of Pt on Mempax glass wafers.

Surface Modification. The platinum nIDEs were first cleaned by rinsing sequentially in dichloromethane, acetone, and ethanol (bare Pt substrates were ultrasonicated in the same solvents for $7 \mathrm{~min}$ each). Platinum surfaces were finally cleaned in $\mathrm{O}_{2}$ plasma (Diener Pico, Diener Electronics, Ebhausen, Germany) for $30 \mathrm{~s}$. After placing the PDMS device on the chip (see Supporting Information, SI-1), the microchannel was filled with a $1 \mathrm{mM}$ AUT solution in ethanol to form a selfassembled thiol monolayer under static incubation at room temperature overnight. Next, the channels were washed with 1 $\mathrm{mL}$ of ethanol, blown dry with $\mathrm{N}_{2}$, filled with neat poly(ethylene glycol) diglycidyl ether (PEGDGE), and left overnight at $40^{\circ} \mathrm{C}$. Afterward, the channels were again rinsed with $1 \mathrm{~mL}$ of ethanol and blown dry with $\mathrm{N}_{2}$. This yields an antibiofouling layer, which was subsequently functionalized with antibodies. Specifically, a capturing anti-EpCAM (C-AE) solution was diluted in sodium phosphate buffer $(\mathrm{pH} \mathrm{8.3)}$ to a final concentration of $25 \mathrm{mg} / \mathrm{mL}$ and injected in the microchannel for overnight incubation. In this step, epoxide groups in the PEGDGE molecules reacted primarily with amines on the lysine residues on the antibody molecules. Prior to the EV capture, unreacted epoxide groups were blocked with a filtered $1 \%$ BSA solution in PBS for $1 \mathrm{~h}$ at room temperature, and the device was rinsed with $1 \mathrm{~mL}$ of PBS.

Characterization of the Surface Functionalization. The antibody functionalization was validated on plain Ptcoated silica substrates before being applied on devices with IDEs (see SI-4 and SI-5). AFM analysis in the air of the dried substrates confirmed the successful and selective capture of tdEVs on the C-AE functionalized Pt surface. Circular objects ( $10 \mathrm{~nm} \pm 1 \mathrm{~nm}$ in height and $0.1-1 \mu \mathrm{m}$ in width) were found in all studied regions of $10 \times 10 \mu \mathrm{m}^{2}$, corresponding to EVs, which have collapsed while drying. In contrast, for negative control samples [(1) no addition of PEGDGE, (2) no antibody conjugation, (3) no incubation with EVs, or (4) incubation with EpCAM-negative EVs derived from the PC3 cell line], no EV was found (Figure SI-2). These conditions were subsequently used to functionalize the IDEs.

\section{ASSOCIATED CONTENT}

\section{S Supporting Information}

The Supporting Information is available free of charge on the ACS Publications website at DOI: 10.1021/acs.nanolett.9b02741.

Description of PDMS device design, fabrication, and operation; tdEV preparation protocol from prostate cancer cell lines; EV preparation protocol from platelets; AFM and XPS results (PDF)

\section{AUTHOR INFORMATION}

\section{Corresponding Authors}

*E-mail: s.legac@utwente.nl.

*E-mail: w.g.vanderwiel@utwente.nl.

ORCID

Pepijn Beekman: 0000-0001-8401-9777

Serge G. Lemay: 0000-0002-0404-3169

Wilfred G. van der Wiel: 0000-0002-3479-8853

\section{Author Contributions}

D.G.M., P.B., S.L.G., and W.G.vdW. conceived the experiments. D.G.M. fabricated the IDEs and performed the electrochemical measurements and the data analysis. P.B. performed the surface functionalization and characterization and the microfluidics. D.G.M. and P.B. wrote the manuscript with contributions from all authors. All authors approved the content of the manuscript.

\section{Author Contributions}

${ }^{\nabla}$ D.G.M. and P.B. contributed equally.

\section{Funding}

This work is part of the Perspectief Program Cancer ID (project no. 14196), which is funded by The Netherlands Organization for Scientific Research (NWO).

\section{Notes}

The authors declare no competing financial interest.

\section{ACKNOWLEDGMENTS}

Prof. Leon Terstappen, Dr. Cees Otto, and Dr. Herman Offerhaus are acknowledged for sharing their invaluable insights. Dr. Sidharam Pujari is acknowledged for his consulting on the antifouling layer. Mr. Tom Stelwagen is also acknowledged for his help with device fabrication.

\section{ABBREVIATIONS}

AFM, atomic force microscopy; ALP, alkaline phosphatase; AUT, amino-undecanethiol; BSA, bovine serum albumin; C$\mathrm{AE}$, capturing anti-EpCAM; CE, counter electrode; CT, computed tomography; CTC, circulating tumor cell; ctDNA, circulating tumor DNA; CV, cyclic voltammogram; EBL, ebeam lithography; EpCAM, epithelial cell adhesion molecule; $\mathrm{EV}$, extracellular vesicle; IDE, interdigitated electrode $K_{\mathrm{d}}$ dissociation constant; LOD, limit-of-detection; MRI, magnetic resonance imaging; nIDE, nanointerdigitated electrode array; NTA, Nanoparticle Tracking Analysis; pAP, para-aminophenol; pAPP, para-aminophenyl phosphate; PBS, phosphate buffered saline; PDMS, poly(dimethylsiloxane); PEGDGE, poly(ethylene glycol) diglycidyl ether; pEV, platelet-derived 
extracellular vesicle; pQI, para-quinone imine; R-AE, reporting anti-EpCAM; RE, reference electrode; SAV-ALP, streptavidinconjugated alkaline phosphatase; tdEV, tumor-derived extracellular vesicle; WE, working electrode; $\mu \mathrm{IDE}$, microinterdigitated electrode array

\section{REFERENCES}

(1) Nanou, A.; Coumans, F. A.; van Dalum, G.; Zeune, L. L.; Dolling, D.; Onstenk, W.; Crespo, M.; Fontes, M. S.; Rescigno, P.; Fowler, G.; et al. Circulating tumor cells, tumor-derived extracellular vesicles and plasma cytokeratins in castration-resistant prostate cancer patients. Oncotarget 2018, 9 (27), 19283.

(2) Alix-Panabières, C.; Pantel, K. Circulating tumor cells: liquid biopsy of cancer. Clin. Chem. 2013, 59 (1), 110-118.

(3) Budd, G. T.; Cristofanilli, M.; Ellis, M. J.; Stopeck, A.; Borden, E.; Miller, M. C.; Matera, J.; Repollet, M.; Doyle, G. V.; Terstappen, L. W.; et al. Circulating tumor cells versus imaging-predicting overall survival in metastatic breast cancer. Clin. Cancer Res. 2006, 12 (21), 6403-6409.

(4) Coumans, F.; Dalum, G.; Terstappen, L. W. M. M. CTC Technologies and Tools. Cytometry, Part A 2018, 93 (12), 11971201.

(5) Vaidyanathan, R.; Soon, R. H.; Zhang, P.; Jiang, K.; Lim, C. T. Cancer diagnosis: from tumor to liquid biopsy and beyond. Lab Chip 2018, 19 (1), 11-34.

(6) Poudineh, M.; Sargent, E. H.; Pantel, K.; Kelley, S. O. Profiling circulating tumour cells and other biomarkers of invasive cancers. Nature Biomedical Engineering 2018, 2 (2), 72.

(7) Yáñez-Mó, M.; Siljander, P. R.-M.; Andreu, Z.; Bedina Zavec, A.; Borràs, F. E.; Buzas, E. I.; Buzas, K.; Casal, E.; Cappello, F.; Carvalho, $\mathrm{J}$. Biological properties of extracellular vesicles and their physiological functions. Journal of extracellular vesicles 2015, 4 (1), 27066.

(8) van Niel, G.; D’Angelo, G.; Raposo, G. Shedding light on the cell biology of extracellular vesicles. Nat. Rev. Mol. Cell Biol. 2018, 19 (4), 213.

(9) Mathieu, M.; Martin-Jaular, L.; Lavieu, G.; Théry, C. Specificities of secretion and uptake of exosomes and other extracellular vesicles for cell-to-cell communication. Nat. Cell Biol. 2019, 21 (1), 9.

(10) Reátegui, E.; Vos, K. E.; Lai, C. P.; Zeinali, M.; Atai, N. A.; Aldikacti, B.; Floyd, F. P.; Khankhel, A.; Thapar, V.; Hochberg, F. H.; et al. Engineered nanointerfaces for microfluidic isolation and molecular profiling of tumor-specific extracellular vesicles. Nat. Commun. 2018, 9 (1), 175.

(11) Koliha, N.; Wiencek, Y.; Heider, U.; Jüngst, C.; Kladt, N.; Krauthäuser, S.; Johnston, I. C.; Bosio, A.; Schauss, A.; Wild, S. A novel multiplex bead-based platform highlights the diversity of extracellular vesicles. J. Extracell. Vesicles 2016, 5 (1), 29975.

(12) Yadav, S.; Boriachek, K.; Islam, M. N.; Lobb, R.; Möller, A.; Hill, M. M.; Hossain, M. S. A.; Nguyen, N. T.; Shiddiky, M. J. An Electrochemical Method for the Detection of Disease-Specific Exosomes. ChemElectroChem 2017, 4 (4), 967-971.

(13) Julich, H.; Willms, A.; Lukacs-Kornek, V.; Kornek, M. Extracellular vesicle profiling and their use as potential disease specific biomarker. Front. Immunol. 2014, 5, 413.

(14) Lobb, R. J.; Lima, L. G.; Möller, A. In Exosomes: key mediators of metastasis and pre-metastatic niche formation, Seminars in cell \& developmental biology; Elsevier: 2017; pp 3-10.

(15) Galindo-Hernandez, O.; Villegas-Comonfort, S.; Candanedo, F.; González-Vázquez, M.-C.; Chavez-Ocaña, S.; Jimenez-Villanueva, X.; Sierra-Martinez, M.; Salazar, E. P. Elevated concentration of microvesicles isolated from peripheral blood in breast cancer patients. Arch. Med. Res. 2013, 44 (3), 208-214.

(16) Coumans, F.; Doggen, C. J. M.; Attard, G.; De Bono, J.; Terstappen, L. W. M. M. All circulating EpCAM+ CK+ CD45objects predict overall survival in castration-resistant prostate cancer. Annals of oncology 2010, 21 (9), 1851-1857.
(17) Hattori, Y.; Shimada, T.; Yasui, T.; Kaji, N.; Baba, Y. Micro-and Nanopillar Chips for Continuous Separation of Extracellular Vesicles. Anal. Chem. 2019, 6514.

(18) Shpacovitch, V.; Hergenroeder, R. Optical and surface plasmonic approaches to characterize extracellular vesicles. A review. Anal. Chim. Acta 2018, 1005, 1-15.

(19) van der Pol, E.; Sturk, A.; van Leeuwen, T.; Nieuwland, R.; Coumans, F.; Mobarrez, F.; Arkesteijn, G.; Wauben, M.; Siljander, P. R.-M.; Sanchez-Lopez, V.; Otero-Candelera, R.; Ramon, L. A.; Dolz, S.; Vila, V.; Mackman, N.; Geddings, J.; Mullier, F.; Bailly, N.; Han, J.Y.; Kwaan, H. C.; Weiss, I. M.; Buzas, E. I.; Pallinger, E.; Harrison, P.; Kraan, J.; Hedley, B. D.; LazoLangner, A.; Enjeti, A.; Norris, P. J.; Paris, C.; Susen, S.; Bonnefoy, A.; Delorme, I.; Chandler, W. L.; Hau, C.; Aass, H. C. D.; Connor, D.; Wu, X.; Dragovic, R.; Uotila, L. M.; Lacroix, R.; Robert, S. Standardization of extracellular vesicle measurements by flow cytometry through vesicle diameter approximation. J. Thromb. Haemostasis 2018, 16 (6), 1236-1245.

(20) Nolan, J. P.; Duggan, E. Analysis of Individual Extracellular Vesicles by Flow Cytometry. In Flow Cytometry Protocols; Springer, 2018; pp 79-92.

(21) Contreras-Naranjo, J. C.; Wu, H.-J.; Ugaz, V. M. Microfluidics for exosome isolation and analysis: enabling liquid biopsy for personalized medicine. Lab Chip 2017, 17 (21), 3558-3577.

(22) Hisey, C. L.; Dorayappan, K. D. P.; Cohn, D. E.; Selvendiran, K.; Hansford, D. J. Microfluidic affinity separation chip for selective capture and release of label-free ovarian cancer exosomes. Lab Chip 2018, 18 (20), 3144-3153.

(23) Im, H.; Lee, K.; Weissleder, R.; Lee, H.; Castro, C. M. Novel nanosensing technologies for exosome detection and profiling. Lab Chip 2017, 17 (17), 2892-2898.

(24) Yukawa, H.; Suzuki, K.; Aoki, K.; Arimoto, T.; Yasui, T.; Kaji, N.; Ishikawa, T.; Ochiya, T.; Baba, Y. Imaging of angiogenesis of human umbilical vein endothelial cells by uptake of exosomes secreted from hepatocellular carcinoma cells. Sci. Rep. 2018, 8 (1), 6765.

(25) Yuana, Y.; Oosterkamp, T.; Bahatyrova, S.; Ashcroft, B.; Garcia Rodriguez, P.; Bertina, R.; Osanto, S. Atomic force microscopy: a novel approach to the detection of nanosized blood microparticles. $J$. Thromb. Haemostasis 2010, 8 (2), 315-323.

(26) Van Der Pol, E.; Hoekstra, A.; Sturk, A.; Otto, C.; Van Leeuwen, T.; Nieuwland, R. Optical and non-optical methods for detection and characterization of microparticles and exosomes. $J$. Thromb. Haemostasis 2010, 8 (12), 2596-2607.

(27) Lee, W.; Nanou, A.; Rikkert, L.; Coumans, F. A.; Otto, C.; Terstappen, L. W.; Offerhaus, H. L. Label-Free Prostate Cancer Detection by Characterization of Extracellular Vesicles Using Raman Spectroscopy. Anal. Chem. 2018, 90 (19), 11290-11296.

(28) Beekman, P.; Enciso-Martinez, A.; Rho, H. S.; Pujari, S. P.; Lenferink, A.; Zuilhof, H.; Terstappen, L. W. M. M.; Otto, C.; Le Gac, S. Immuno-capture of extracellular vesicles for individual multi-modal characterization using AFM, SEM and Raman spectroscopy. Lab Chip 2019, 19, 2526.

(29) Zhang, P.; Zhou, X.; He, M.; Shang, Y.; Tetlow, A. L.; Godwin, A. K.; Zeng, Y. Ultrasensitive detection of circulating exosomes with a 3D-nanopatterned microfluidic chip. Nature Biomedical Engineering 2019, 3, 438.

(30) Boriachek, K.; Masud, M. K.; Palma, C.; Phan, H.-P.; Yamauchi, Y.; Hossain, M. S. A.; Nguyen, N.-T.; Salomon, C.; Shiddiky, M. J. Avoiding Pre-Isolation Step in Exosome Analysis: Direct Isolation and Sensitive Detection of Exosomes Using GoldLoaded Nanoporous Ferric Oxide Nanozymes. Anal. Chem. 2019, 91, 3827.

(31) Huang, R.; He, L.; Xia, Y.; Xu, H.; Liu, C.; Xie, H.; Wang, S.; Peng, L.; Liu, Y.; Liu, Y.; et al. A Sensitive Aptasensor Based on a Hemin/G-Quadruplex-Assisted Signal Amplification Strategy for Electrochemical Detection of Gastric Cancer Exosomes. Small 2019, 15 (19), 1900735.

(32) Schasfoort, R. B.; Andree, K. C.; van der Velde, N.; van der Kooi, A.; Stojanović, I.; Terstappen, L. W. Interpolation method for 
accurate affinity ranking of arrayed ligand-analyte interactions. Anal. Biochem. 2016, 500, 21-23.

(33) Hermanson, G. T. Bioconjugate Techniques, 2nd ed.; Elsevier Inc., 2008.

(34) Sridhar, A.; van den Berg, A.; Le Gac, S. Non-Invasive Monitoring of Osteogenic Differentiation on Microtissue Arrays under Physiological Conditions Using Scanning Electrochemical Microscopy. Electroanalysis 2014, 26, 1881-1885.

(35) Obeid, S.; Sung, P.-S.; Le Roy, B.; Chou, M.-L.; Hsieh, S.-L.; Elie-Caille, C.; Burnouf, T.; Boireau, W. NanoBioAnalytical characterization of extracellular vesicles in 75-nm nanofiltered human plasma for transfusion: A tool to improve transfusion safety. Nanomedicine 2019, 20, 101977.

(36) Rho, H. S.; Yang, Y.; Veltkamp, H.; Gardeniers, H. Direct Delivery of Reagents from a Pipette Tip to a PDMS Microfluidic Device. In Chips and Tips; RSC Blogs, 2015.

(37) Baggerman, J.; Smulders, M. M.; Zuilhof, H. Romantic Surfaces: A Systematic Overview of Stable, Biospecific, and Antifouling Zwitterionic Surfaces. Langmuir 2019, 35 (5), 10721084. 